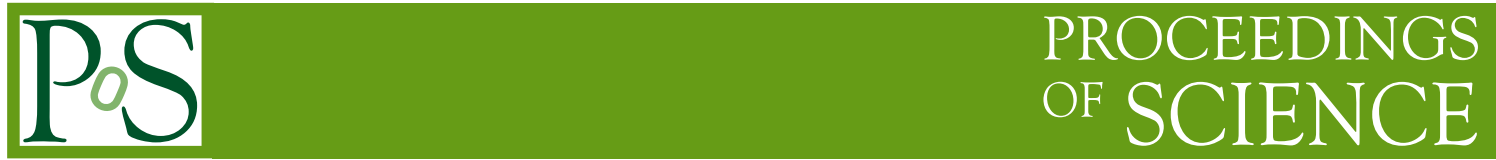

\title{
Phases of many flavors QCD: lattice results
}

\section{Albert Deuzeman}

Albert Einstein Center for Fundamental Physics - University of Bern, Switzerland

E-mail: deuzeman@itp.unibe.ch

\section{Maria Paola Lombardo*, Kohtaroh Miura}

INFN-Laboratori Nazionali di Frascati, I-00044, Frascati (RM), Italy

E-mail: lombardo@lnf.infn.itmiura@lnf.infn.it

\section{Tiago Nunes da Silva, Elisabetta Pallante}

Centre for Theoretical Physics, University of Groningen, 9747 AG, Netherlands

Email: e.pallante@rug.nl tiagoj.nunes@gmail.com

This note is based on our recent results on QCD with varying number of flavors of fundamental fermions. Topics include unusual, strong dynamics in the preconformal, confining phase, the physics of the conformal window and the role of ab-initio lattice simulations in establishing our current knowledge of the phases of many flavor QCD.

Xth Quark Confinement and the Hadron Spectrum

8?12 October 2012

TUM Campus Garching, Munich, Germany

\footnotetext{
${ }^{*}$ Speaker.
} 


\section{Phases of Strong Interactions from the Lattice}

While strong interactions spontaneously break chiral symmetry in ordinary QCD at zero temperature, chiral symmetry is realised either at high temperatures - in the so called quark-gluon plasma (QGP) phase - and at zero temperature for a large number of flavours $N_{f}>N_{f}^{c}$ [1]. In the latter case, the theory is expected to become not only chirally but also conformally invariant, due to the emergence of an infra-red fixed point (IRFP) for $N_{f}>N_{f}^{c}$ which prevents the coupling from growing large enough to break chiral symmetry. The main phenomena under scrutiny are non-perturbative : only lattice analysis affords the possibility of ab-initio studies which are indeed being carried out by many groups[2].

In our studies we have identified two main themes of interest: the physics of the nearconformal window, and the observation of the conformal window. I will discuss them in the light of the results we have obtained in recent years.

\section{Near-conformal : continuum and lattice}

In the near-conformal region[3, 4] we are mostly concerned with precursory effects of conformality when approaching $N_{f}^{c}$ from the QCD side. Model studies suggest three possible scenarios: an essential singularity a la Miransky-Yamawaki [5] $1 / \xi=\exp \left(-\frac{\pi}{2} \varepsilon \sqrt{\left|N f-N_{f}^{c}\right|}\right)$; a power law conventional behaviour [6] $1 / \xi=K\left|N_{f}-N_{f}^{c}\right|^{-1 / \theta}$; and a 'jump' into conformality [7]. In the two first cases the approach to conformality is continuous, and one is likely to observe precursory effects. The distinction might not be so clear-cut and combinations of the various behaviours can be observed as well, as in a weak first order transition where an apparent power law behaviour ends with a small jump at the true critical point.

How do we distinguish a QCD-like dynamics from a more exotic one? In either cases chiral symmetry is broken. Gauge dynamics, however, can be significantly different. The coupling might show a so-called walking behaviour: at a variance with the ordinary running, which is regulated by one unique scale $\Lambda$, a walking behaviour is characterized by two different scales : above the UV scale $\Lambda_{U V}$ the coupling runs towards asymptotic freedom, and below the IR scale $\Lambda_{I R}$ it runs towards confinement, being nearly constant (walking) in between.

In short, we can observe walking either by assessing the existence of two different scales $\Lambda_{U V}$ and $\Lambda_{I R}$ and by observing a pre-critical behaviour when approaching a critical number of flavor $N_{f}^{c}$. Note that $1 / \xi$ in the above expressions denotes any physical quantity with a mass dimension, which includes the critical temperature $T_{c}\left(N_{f}\right)$. This observation paves the way of a study of conformality based on the analysis of a thermal system.

\section{Towards conformality : continuum analysis from lattice results}

A first natural way to highlight a pseudocritical behaviour approaching conformality is based on the analysis of the $N_{f}$ dependence of the critical temperature [3,4]. On a lattice the critical temperature is given by

$$
T_{c} \equiv \frac{1}{a\left(\beta_{\mathrm{L}}^{\mathrm{c}}\right) \cdot N_{t}}
$$



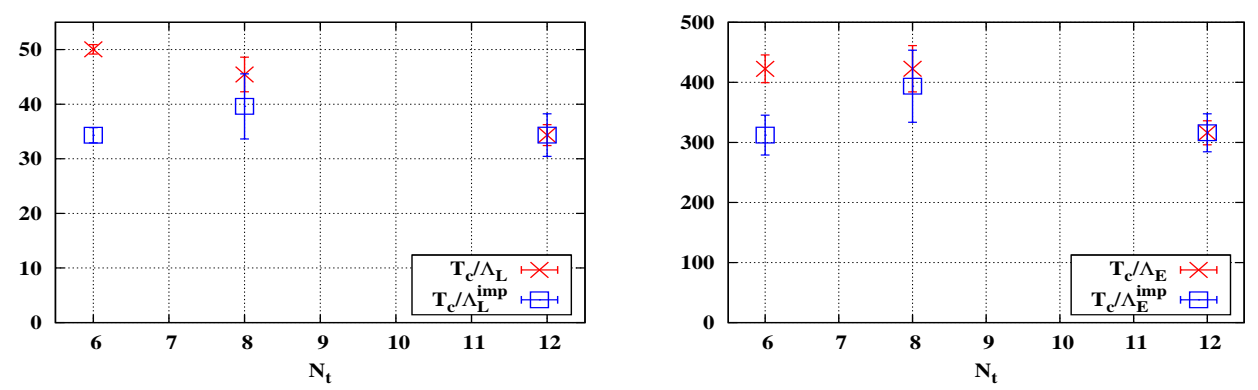

Figure 1: Scaling at $N_{f}=8$ from the $N_{t}$ dependence of the normalised critical temperature. Left: The bare lattice scheme results. The red symbol $\times$ shows $T_{c} / \Lambda_{\mathrm{L}}$, and the blue $\square$ symbols represent $T_{c} / \Lambda_{\mathrm{L}}^{\mathrm{imp}}$. Right: The E-scheme results. The red symbol $\times$ shows $T_{c} / \Lambda_{\mathrm{L}}$, and the blue $\square$ symbols represent $T_{c} / \Lambda_{\mathrm{E}}^{\mathrm{imp}}$

Table 1: Summary of $T_{c} / \Lambda_{\mathrm{L}}$ and $T_{c} / \Lambda_{\mathrm{L}}^{\mathrm{imp}}$ for various $\left(N_{f}, N_{t}\right)$. The first (second) line at fixed $\left(N_{f}, N_{t}\right)$ shows the value of $T_{c} / \Lambda_{\mathrm{L}}\left(T_{c} / \Lambda_{\mathrm{L}}^{\mathrm{imp}}\right)$

\begin{tabular}{|c|c|c|c|c|c|}
\hline$N_{f} \backslash N_{t}$ & 4 & 6 & 8 & 12 & \\
\hline \multirow[t]{2}{*}{0} & $18.11 \pm 0.65$ & $18.21 \pm 0.91$ & $16.56 \pm 0.71$ & & \\
\hline & $16.29 \pm 0.75$ & $17.81 \pm 1.02$ & $16.56 \pm 0.78$ & & \\
\hline \multirow[t]{2}{*}{4} & $21.99 \pm 1.04$ & $19.98 \pm 0.95$ & $17.12 \pm 2.43$ & - & $-\quad-$ \\
\hline & $16.56 \pm 1.44$ & $18.67 \pm 1.38$ & $17.12 \pm 3.41$ & & \\
\hline \multirow[t]{2}{*}{6} & $25.41 \pm 1.43$ & $25.33 \pm 1.43$ & $22.94 \pm 1.29$ & $22.30 \pm 2.52$ & \\
\hline & $21.66 \pm 1.64$ & $23.87 \pm 1.58$ & $22.21 \pm 1.40$ & $22.30 \pm 2.66$ & \\
\hline \multirow[t]{2}{*}{8} & - & $50.05 \pm 0.87$ & $47.06 \pm 3.28$ & $34.34 \pm 1.91$ & \\
\hline & - & $34.32 \pm 1.40$ & $42.67 \pm 6.33$ & $34.34 \pm 3.90$ & \\
\hline
\end{tabular}

which becomes independent on $N_{t}$ close to the continuum limit $a \rightarrow 0$. One way to convert to physical units relies on the normalised critical temperature $T_{c} / \Lambda_{\mathrm{L} / \mathrm{E}}$ where $\Lambda_{\mathrm{L}}\left(\Lambda_{\mathrm{E}}\right)$ represents the lattice (E-scheme) Lambda-parameter defined in the two-loop perturbation theory with or without a renormalisation group inspired improvement. The results for $T_{c} / \Lambda_{\mathrm{L} / \mathrm{E}}$ for the $N_{f}=8$ theory $[8,4]$ are shown in Fig. 1, and the full set of results is collected in Table 1.

From Table 1 we can read the results for $T_{c} / \Lambda$ as a function of $N_{f}$ in different schemes $\left(\Lambda=\Lambda_{\mathrm{L}}\right.$ or $\Lambda_{\mathrm{E}}$ ), which consistently show an increase with $N_{f}$. This indicates that $\Lambda_{\mathrm{L} / \mathrm{E}}$ vanishes faster than $T_{c}$ upon approaching the critical number of flavour. Within the various uncertainties discussed here, this can be taken as a qualitative indication of scale separation close to the critical number of flavors.

We can now further investigate the vanishing of the critical temperature. To this end, we have to face an apparent puzzle : $T_{c} / \Lambda$ increases as a function of $N_{f}$ ! This however, as mentioned, can be understood in terms of scale separation with $\Lambda$ vanishing faster than $T_{c}$. To see the vanishing of $T_{c}$ at $N_{f}^{c}$ we need to replace $\Lambda$ with a UV scale. To do this, we devised a 'baby-version' of the scale setting procedure in the potential scheme. In that scheme. one fixes a value for the renormalised 

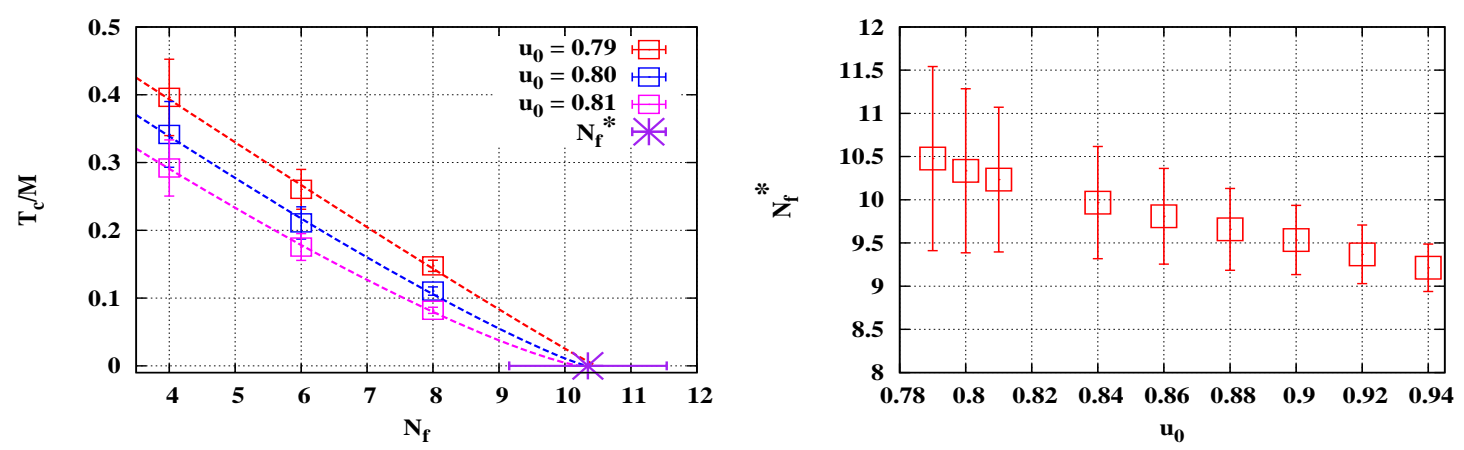

Figure 2: Left: The $N_{f}$ dependence of $T_{c} / M$ where $M$ is determined to be a UV scale corresponding to $u_{0}=0.79$ (red box), 0.80 (blue $\bigcirc$ ), and 0.81 (magenta triangle). Right: The $u_{0}$ dependence of $N_{f}^{c}$. The three data in the left side are determined within the condition $M\left(g_{\mathrm{L}}^{\text {ref }}\right) \lesssim a^{-1}\left(g_{\mathrm{L}}^{\mathrm{c}}\right)$, while for the others $M\left(g_{\mathrm{L}}^{\text {ref }}\right)$ exceeds the lattice cutoff. This more robust procedure confirms our early results, and should be ultimately confirmed by use a rigorous lattice scale setting which is in progress [9].

coupling $\bar{g}$ and $\bar{g}^{2} \propto r_{X}^{2} F\left(r_{X}\right)$ sets a scale $r_{X}^{-1}$. We used our plaquette values to define a coupling at the scale of the lattice spacing, and we set a common UV scale for diffeent theories by imposing a constant value for the coupling (or, equivalently, for the plaquette). In short, we use our $u_{0}=\langle P\rangle^{\frac{1}{4}}$ to define $\bar{g}$, and $u_{0}=X$ is regarded as the analog of the potential scheme scale setting. For this procedure to work, the coupling should be weak enough to be in the UV region, but also large enough to avoid major lattice artifacts. We have checked - the interested reader is referred to Ref. [4] for details - that it is possible to meet these requirements and define consistently an UV coupling over a rather large set of possible choices of $X$. The results are shown in Fig. 2

An alternative analysis stems from a discussion presented in Ref. [10]. Since the critical temperature is zero in the conformal phase, the thermal critical coupling $g_{\mathrm{T}}^{\mathrm{c}}$ should equal a zero temperature critical coupling $g^{c}$ when $N_{f}=N_{f}^{c}$. One possibility is to use the Schwinger-Dyson estimate for $g_{c}$ [11]. In this case, the lower edge of the conformal window $N_{f}^{c}$ is defined by the condition $g_{\mathrm{T}}^{\mathrm{c}}\left(N_{f}^{c}\right)=g_{\mathrm{SD}}^{\mathrm{c}}\left(N_{f}^{c}\right)$ We then estimate the intersection of $g_{\mathrm{T}}^{\mathrm{c}}$ and $g_{\mathrm{SD}}^{\mathrm{c}}$ - hence the onset of the conformal window as well as the IRFP coupling at $N_{f}^{c}$ at $\left(g^{c}, N_{f}^{c}\right)=(2.79,13.2) \pm(0.13,0.6)$. One second possibility is to match $g_{\mathrm{T}}^{\mathrm{c}}\left(N_{f}^{c}\right)$ and the coupling at IRFP ( $\left.g^{\mathrm{IRFP}}\right)$ [12]. We can then locate the intersection of $g_{\mathrm{T}}^{\mathrm{c}}$ and $g_{41}^{\mathrm{IRFP}}$ and obtain $\left(g^{c}, N_{f}^{c}\right)=(2.51,11.8) \pm(0.15,0.9)$. In Fig. 3, we show $g^{\mathrm{IRFP}}$ and $g_{\mathrm{SD}}^{\mathrm{c}}$ alongside with the numerical results for $g_{\mathrm{T}}^{\mathrm{c}}$, as well as the estimates for the IRFP.

\section{Towards conformality : Lattice analysis}

The emergence of the conformal window can also read-off directly from the lattice data themselves. Consider the phase diagram in the space spanned by the bare coupling $g_{\mathrm{L}}$ and the number of flavor $N_{f}$, and the (pseudo)critical thermal lines which connect the lattice (pseudo)critical couplings for a fixed $N_{t}$. Based on the properties of the step scaling function in the vicinity of a IRFP [13], it is easy to convince ourselves that the critical number of flavor $N_{f}^{c}$ can be identified with the crossing point the pseudocritical thermal lines obtained for various $N_{t}$ 's 


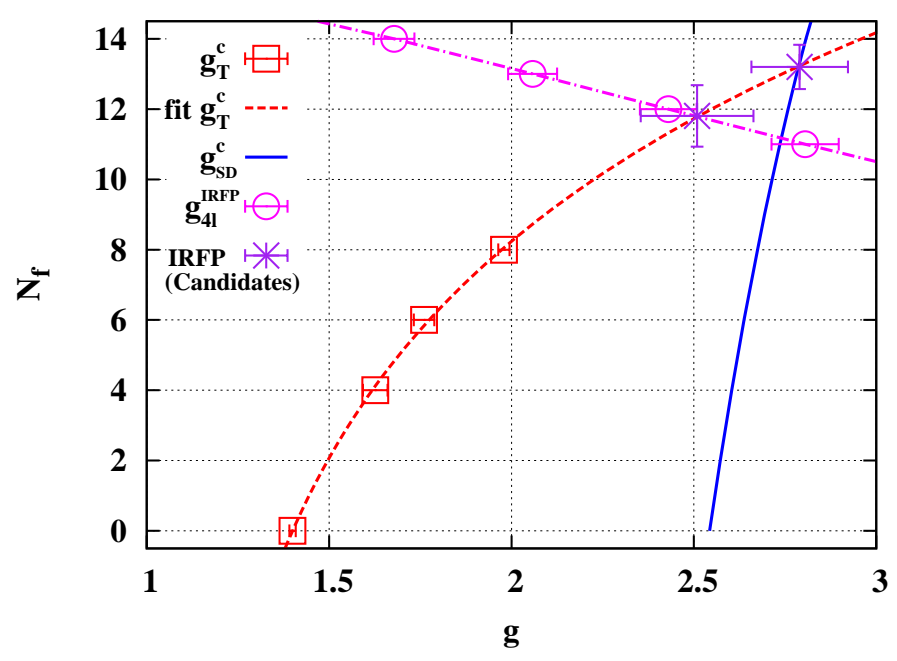

Figure 3: The thermal critical coupling (red $\square$ ) and the fit for them (dashed red line) and the values of the zero temperature couplings in the conformal phase from different estimates, see text for details. At the critical number of flavour the thermal critical coupling should equal the critical coupling associated with the IRFP.

To demonstrate this procedure, we consider the pseudocritical lines obtained for $N_{t}=6$ and $N_{t}=12$ as shown in Fig. 4. Note their positive slope: the lattice critical coupling $g_{\mathrm{L}}{ }^{\mathrm{c}}$ is an increasing function of $N_{f}$. Interestingly, the slope decreases with increasing $N_{t}$, which allows for a crossing point at a larger $N_{f}$. Thus, we estimate the intersection at $\left(g_{\mathrm{L}}^{\mathrm{c}}, N_{f}^{c}\right)=(1.79 \pm 0.12,11.1 \pm 1.6)$.

\section{Inside the conformal window: continuum results}

Conformal symmetry implies chiral symmetry. As we are seeking evidence for conformality in QCD, a natural strategy is to establish whether the theory realizes chiral symmetry at zero temperature $[14,15]$.

A direct observation of chiral symmetry can indeed be attempted: this means extrapolating the chiral condensate to the chiral limit. Of course there are obvious numerical limitations : firstly, the functional form depends on the realization of chiral symmetry; second, the extrapolated value is affected by a residual error. So all one can try is to compare side by side the quality of the extrapolations carried out with different analytic ansätze. While for a small number of flavors such procedure would unambiguously indicate the breaking of chiral symmetry, when $N_{f}$ grows large say above $N_{f}=8$ the results become more ambigous - on which point everyone agrees - with some groups favoring chiral symmetry restoration (hence conformality), and other chiral breaking.

The analysis of the spectrum might offer a more realiable guidance : it has been noted in the past that one can devise robust signatures of chiral symmetry based on the analysis of the spectrum results. One first significant spectrum observable is the ratio $m_{\pi} / m_{\rho}$, between the mass of the lightest pseudoscalar state (pion) $m_{\pi}$ and the mass of the lightest vector state (rho) $m_{\rho}$. In QCD at zero temperature, chiral symmetry is spontaneously broken and the pion is the (pseudo)Goldstone boson of the broken symmetry, implying that its mass will behave as $m_{\pi} \sim \sqrt{m}$. 


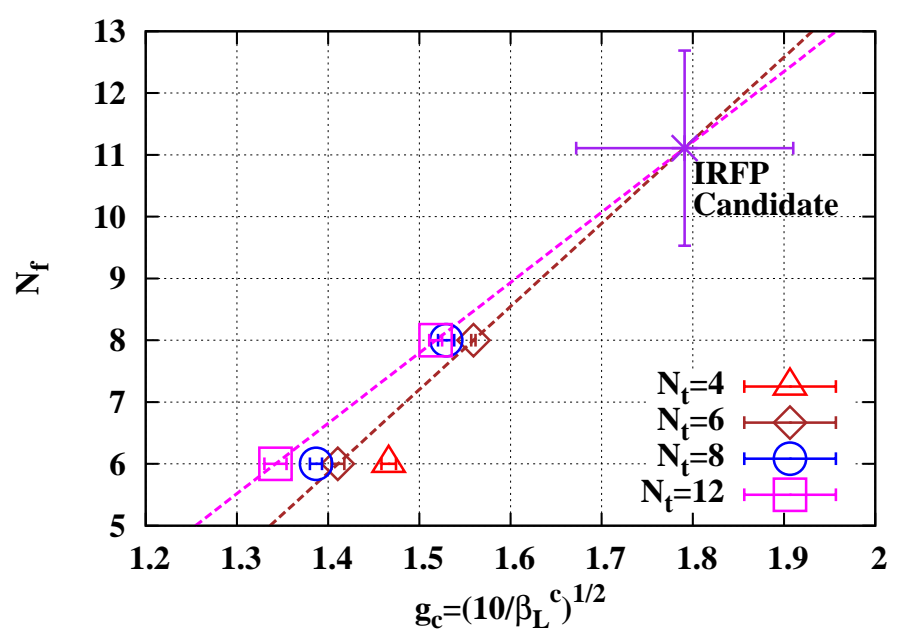

Figure 4: (Pseudo) critical values of the lattice coupling $g_{\mathrm{L}}^{\mathrm{c}}=\sqrt{10 / \beta_{\mathrm{L}}}{ }^{\mathrm{c}}$ for theories with $N_{f}=0,4,6,8$ and for several values of $N_{t}$ in the Miransky-Yamawaki phase diagram. We have picked up $g_{\mathrm{L}}^{\mathrm{c}}$ at $N_{f}=6$ and 8 , and considered "constant $N_{t}$ " lines with $N_{t}=6,12$. If the system is still described by one parameter beta-function in this range of coupling, the IRFP could be located at the intersection of the fixed $N_{t}$ lines .

Within the conformal window chiral symmetry is restored in the continuum limit.

At the IRFP and at infinite volume, the quark mass dependence of all hadron masses in the spectrum is governed by conformal symmetry: at leading order in the quark mass expansion all masses follow a power-law with common exponent determined by the anomalous dimension of the fermion mass operator at the IRFP. Hence we expect a constant ratio. Away from the IRFP, for sufficiently light quarks and finite lattice volumes, the universal power-law dependence receives corrections, due to the fact that the theory is interacting but no longer conformal. The behaviour of the ratio is demonstrated in Fig. 5: a conformal scenario seems favoured in the range of masses we are exploring. Note that the $m_{\pi} / m_{\rho}$ ratio should go to zero in the chiral limit in the broken phase, and to a constant value if chiral symmetry is restored.

Analgous conclusions can be drawn from the inspection of the so called Edinburgh plot (6). The difference with the case of ordinary QCD is indeed striking. The modest scattering of the data points could be ascribed to the deviation from a perfect power law as discussed above. It would then be of interest to repeat the same plot for different couplings : at the IRFP it should indeed reduce to a point.

\section{Inside the conformal phase : lattice}

If we were to use a perfect action the conformal phase discussed above would extend all the way till the infinite coupling limit. With a naive action instead chiral symmetry appears to be always broken in the strong coupling limit, at least till $N_{f}$ is not too large. An obvious consequence of this is the occurrence of a strong coupling zero temperature transition - a bulk transition - within the conformal window. The role of improvement in this case is really dramatic! A perfect action would destroy a phase transition. No suprise, of course: these are strong coupling phenomena 


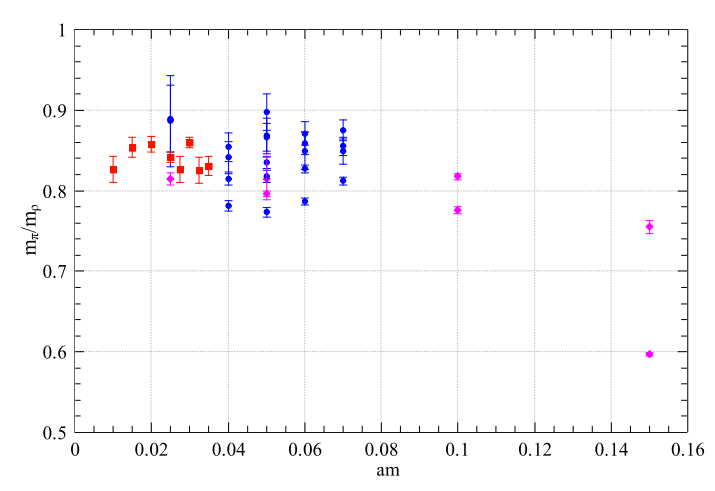

Figure 5: Ratio $m_{\pi} / m_{\rho}$ as a function of the bare quark mass for all existing data for $N_{f}=12$, and $N_{f}=16$ : $N_{f}=12$ data from [16] (red squares), $N_{f}=12$ data from this work and $\beta_{L}=3.8,3.9,4.0$ (blue circles), $N_{f}=16$ data from [17] (magenta diamonds).

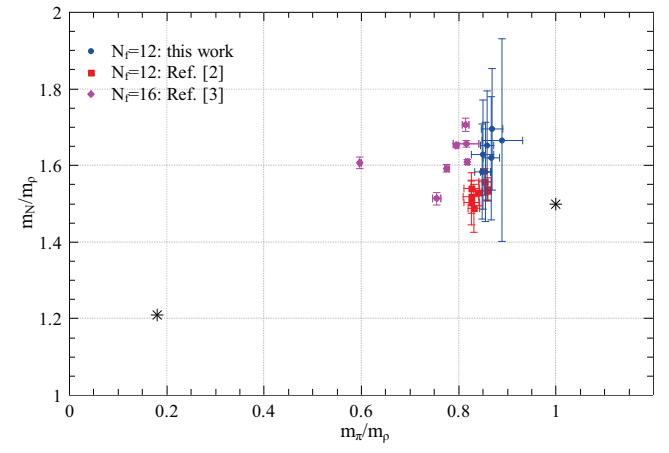

Figure 6: Edinburgh plot: $N_{f}=12$ data from [16] (red squares), $N_{f}=12$ data from this work and $\beta_{L}=$ 3.8,3.9 (blue circles), $N_{f}=16$ data from [17] (magenta diamonds). The QCD physical point (black star, leftmost) and the heavy quark limit (free theory) point (black star, rightmost) are shown.

taking place away from the continuum limit, hence extra terms in the actions which are irrelevant in the continuum might well become relevant.

But then, how would an ordinary improved action (as opposed to a perfect action) affect the phase transition? The evidence we have so far is in this case $[19,18]$ the bulk transition moves towards stronger coupling (consistently with the fact that it will eventually disappear with a perfect action), and a second transition develops. Among these two transitions we have a phase with an unusual realization of chiral symmetry, observed also in other studies[20].

From the perspective of the analysis of continuum many flavor QCD these observations are just due to a peculiar form of lattice artifacts. Bulk transitions are however interesting for several reasons including fundamental QFT questions like the existence of an interacting, non-trivial UV fixed point in four dimension away from the perturbative domain as well as modeling of condensed matter systems, such as graphene, and the new phases discussed here might well be of interest in these contexts.

\section{Summary}

In brief summary, we have studied the physics of the near-conformal window and observed a likely scale separation for $N_{f}>6$. We have developed suitable extrapolation techniques and estimate in several different ways the critical number of flavors to be

$$
N_{f}^{c} \sim \begin{cases}11.1 \pm 1.6 & \text { (from the vanishing thermal scaling of } \beta_{\mathrm{L}}^{\mathrm{c}} \text { ) } \\ 12.5 \pm 1.6 & \text { (from the approach of } \left.g_{\mathrm{T}}^{\mathrm{c}} \text { to } g_{\mathrm{SD}}^{\mathrm{c}} \text { and } g_{41}^{\mathrm{IRFP}}\right) \\ 10.4 \pm 1.2 & \text { (from the vanishing of } T_{c} / M \text { with } M \text { a UV scale) }\end{cases}
$$

Although these estimates obviously lack precision, they highlight in a simple way the emergence of conformality. 
In the conformal window we have shown how the spectrum analysis can give information on the realization of chiral symmetry, and we have discussed features of the strong coupling regime which might be of interest when modeling a rather a wide class of phenomena including phase transitions in condensed matter.

There are several directions in which this work can, and hopefully will be extended: a more rigourours scale setting in the preconformal region is on the way. The IRFP should be clearly observed, and we are aiming at doing it working within the analytically tractable $N_{f}=16$ model. At a more theoretical level, we hope that this analysis will help clarifying the behaviour of the anomalus dimension in the vicinity and away from the IRFP.

More generally, the interplay of the cold conformal window phase with thermal QCD and the physics of the Quark Gluon Plasma is an interesting, still largely unexplored, field of research which we hope to further pursue in a near future.

\section{References}

[1] F. Sannino, these proceedings;

[2] See J. Giedt, PoS LATTICE 2012 (2012) 006 for the most recent lattice review, and please consult the cited papers for a comprehensive set of references;

[3] K. Miura, M. P. Lombardo and E. Pallante, Phys. Lett. B 710 (2012) 676;

[4] K. Miura and M. P. Lombardo, arXiv:1212.0955 [hep-lat], Nucl. Phys. B, in press;

[5] V. A. Miransky and K. Yamawaki, Phys. Rev. D 55 (1997) 5051 [Erratum-ibid. D 56 (1997) 3768];

[6] J. Braun, C. S. Fisher, H. Gies, Phys. Rev. D84 (2011) 034045;

[7] O. Antipin, M. Mojaza and F. Sannino, arXiv:1208.0987 [hep-ph]

[8] A. Deuzeman, M. P. Lombardo and E. Pallante, Phys. Lett. B 670 (2008) 41.

[9] A. Deuzeman, M. P. Lombardo, K. Miura, E. Pallante, and T. N. da Silva, in progress;

[10] J. Liao, E. Shuryak and E. Shuryak, Phys. Rev. Lett. 109, 152001 (2012);

[11] T. Appelquist, A. Ratnaweera, J. Terning and L. C. R. Wijewardhana, Phys. Rev. D 58 (1998) 105017.

[12] T. A. Ryttov and R. Shrock, Phys. Rev. D 86 (2012) 085005;

[13] A. Hasenfratz, Phys. Rev. Lett. 108 (2012) 061601;

[14] A. Deuzeman, M. P. Lombardo and E. Pallante, Phys. Rev. D 82 (2010) 074503;

[15] A. Deuzeman, M. P. Lombardo and E. Pallante, PoS LATTICE 2011 (2011) 083;

[16] Z. Fodor, K. Holland, J. Kuti, D. Nogradi and C. Schroeder, Phys. Lett. B703 (2011) 348-358.

[17] P. H. Damgaard, U. M. Heller, A. Krasnitz and P. Olesen, Phys. Lett. B 400 (1997) 169

[18] A. Deuzeman, M. P. Lombardo, T. N. da Silva and E. Pallante, arXiv:1209.5720 [hep-lat], Phys. Lett. $\mathrm{B}$, in press

[19] T. N. da Silva and E. Pallante, arXiv:1211.3656 [hep-lat];

[20] A. Cheng, A. Hasenfratz and D. Schaich, Phys. Rev. D 85 (2012) 094509 